\section{Efeitos do treinamento físico aeróbico contínuo e resistido na função endotelial mediada pelo óxido nítrico: uma revisão sistemática}

\section{Effects of continuous aerobic and resistance exercise trainings on the nitric oxide-mediated vascular function: a systematic review}

\author{
Aurora Corrêa Rodrigues ${ }^{1}$ \\ Renata Aparecida Rodrigues de Oliveira ${ }^{1}$ \\ João Carlos Bouzas Marins ${ }^{2}$ \\ Antônio José Natali ${ }^{2}$
}

\section{Resumo}

O objetivo desta revisão sistemática foi examinar evidências de que treinamentos físicos com diferentes tipos de exercícios influenciam o papel do óxido nítrico (NO) derivado do endotélio na função vascular em indivíduos saudáveis. Foi realizada uma pesquisa nas bases de dados $\mathrm{Me}$ dline e Science Direct sobre artigos publicados entre janeiro de 1997 e maio de 2012. Foram incluídos estudos originais sobre os efeitos do treinamento físico na função vascular em humanos saudáveis. Para análise, os estudos foram divididos em treinamentos com exercícios resistidos, aeróbicos contínuos ou combinados (aeróbico contínuo e resistido). Treze artigos atenderam aos critérios de inclusão/exclusão, sendo cinco estudos com exercícios resistidos, sete com aeróbicos contínuos e um com combinados. Dentre os estudos com exercícios aeróbicos, três observaram aumento no fluxo sanguíneo, um observou melhora na condutância vascular, um demonstrou melhora no tônus vascular e dois demonstraram aumento na concentração de nitrito/nitrato. Com exercícios resistidos, cinco estudos observaram aumento no fluxo sanguíneo e dois reportaram aumento da condutância vascular. $\mathrm{O}$ exercício aeróbico realizado após o resistido aumenta o fluxo sanguíneo, parece diminuir a rigidez arterial e a velocidade da onda de pulso. Concluiu-se que treinamentos físicos com exercícios aeróbicos contínuos ou resistidos influenciam beneficamente o papel do NO derivado do endotélio na função vascular.

\section{Palavras-chave}

Atividade física; Endotélio; Exercício; Vasodilatação; Óxido nítrico.

\begin{abstract}
This systematic review was undertaken to examine evidences that exercise training using different types of exercise influences the roll of the NO derived from the endothelium in the vascular function in healthy individuals. Two electronic databases, PubMed e Science Direct, were searched to identify original articles on this issue with human beings published from January 1997 to May 2012. Thirteen articles met the inclusion/exclusion criteria. For analysis the articles were divided into aerobic (seven), resistance (five) and combined (aerobic + resistance) exercise trainings (one). Amongst the studies using aerobic exercises, three reported increased blood flow, one observed improved vascular conductance, one demonstrated improved vascular tone and two showed increased nitrite/nitrate concentrations. Five studies using resistance exercise reported augmented blood flow and two studies demonstrated increased vascular conductance. Aerobic exercise performed after resistance exercise increases blood flow and reduces arterial stiffness and pulse wave velocity. In conclusion, training programs using either aerobic or resistance exercise trainings influences positively the roll of the NO derived from the endothelium on the vascular function.
\end{abstract}

\section{Keywords}

Physical activity; Endothelium; Exercise; Vasodilation; Nitric oxide.
Rev Bras Ativ Fis Saúde p. 286-297 DOI:

http://dx.doi.org/10.12820/rbafs.v.18n3p286

1 Mestranda em Educação Física pela Universidade Federal de Viçosa - Minas Gerais, Brasil.

2 Professor Associado da Universidade Federal de Viçosa - Minas Gerais, Brasil. 


\section{INTRODUÇÃO}

O óxido nítrico (NO) é uma molécula de sinalização gasosa e lipossolúvel que contribui para a regulação de uma ampla variedade de processos no sistema cardiovascular, nervoso e imune ${ }^{1}$. Nas células do endotélio o NO é sintetizado pela enzima óxido nítrico sintase endotelial (eNOS), a partir do seu precursor o aminoácido $\mathrm{L}$-arginina ${ }^{2,3}$. Por ser lipossolúvel o NO rapidamente cruza o espaço intersticial e difunde-se nas células do músculo liso vascular, onde uma cascata de sinalização promove o relaxamento destas células ${ }^{4}$. $\mathrm{O}$ NO tem meia-vida de poucos segundos em um ambiente aquoso, podendo ser maior que 15 segundos em ambientes com menores concentrações de oxigênio ${ }^{5}$. O estudo de Furchgott e Zawadzki ${ }^{6}$ foi o primeiro a demonstrar que o relaxamento dos vasos sanguíneos, induzido por acetilcolina, requer a presença de células endoteliais. Em 1987, o NO foi identificado como um fator relaxante dos vasos sanguíneos ${ }^{7,8}$.

O NO e o superóxido são radicais livres primários produzidos nas células, ambos são reativos e podem prontamente reagir para formar uma série de outras espécies reativas de oxigênio e de nitrogênio (ROS/RNS) ${ }^{9}$. Apesar de diversos tecidos produzirem ROS, o músculo esquelético é o maior produtor de radicais livres (RL) e de ROS durante o exercício, com a contração muscular são produzidos superóxido e $\mathrm{NO}^{9}$. O superóxido, em altas concentrações, pode reagir com o $\mathrm{NO}$ para formar o peroxynitrito, um potente oxidante ${ }^{10}$, que participa do processo de disfunção endotelial e da redução na biodisponibilidade de $\mathrm{NO}^{11}$.

A fim de eliminar as ROS/RNS e manter a homeostase redox, as células são munidas de antioxidantes enzimáticos e não-enzimáticos ${ }^{12}$. Os antioxidantes enzimáticos, como a SOD, são os que mais contribuem para a capacidade antioxidante total ${ }^{13}$. Estes reagem com os RL e as ROS, minimizando suas ações e, consequentemente, retardando ou prevenindo o estresse oxidativo ${ }^{14}$.

Embora o exercício agudo gere um aumento transitório no estresse oxidativo, pela produção acelerada de ROS, o treinamento físico pode aumentar a disponibilidade de defesas antioxidantes enzimáticas no tecido vascular ${ }^{11}$. $\mathrm{O}$ aumento das ROS durante o exercício leva a um favorável aumento na atividade das enzimas antioxidantes, devido aos RL produzidos durante a contração muscular atuarem como moléculas sinalizadoras que estimulam a expressão gênica e, consequentemente, o aumento da produção de enzimas antioxidantes ${ }^{14}$. $\mathrm{O}$ treinamento de corrida, em modelo animal, foi capaz de reduzir a produção de ROS e aumentar a atividade da $\mathrm{SOD}^{15}$. A reação entre a $\mathrm{SOD}$ e o superóxido produz o peróxido de hidrogênio, um agente oxidante menos ofensivo do que o peroxynitrito ${ }^{10}$, o qual está envolvido no aumento da expressão da eNOS ${ }^{16}$.

O exercício físico induz adaptações fisiológicas ao sistema cardiovascular, sendo uma delas o aumento do fluxo sanguíneo para o músculo esquelético durante o exercício $^{17}$. Esse aumento do fluxo sanguíneo gera estresse de cisalhamento nas paredes dos vasos sanguíneos, que estimula o endotélio a liberar o $\mathrm{NO}^{18}$. A produção do $\mathrm{NO}$ regulada pela ativação da eNOS ocorre em resposta ao aumento da concentração de cálcio no citoplasma das células endoteliais. Os íons cálcio ligam-se a calmodulina a fim de ativar a eNOS ${ }^{12}$. Ao difundir-se nas células do músculo liso presentes no vaso sanguíneo o NO liga-se a guanilato ciclase solúvel (GCs) formando o monofosfato de guanosina ciclico $(\mathrm{GMPc})^{19}$. Este segundo mensageiro reduz a contratilidade destas células por antagonizar a liberação do cálcio do retículo sarcoplasmático e a sua entrada através dos canais de cálcio, e por inibir o processo de contração ativado pelo cálcio 4 . Esse processo promove o relaxamento do músculo liso e a vasodilatação dos vasos ${ }^{19}$. 
Repetidas exposições ao estresse de cisalhamento decorrente do treinamento físico pode resultar na adaptação endotelial, que inclui o aumento na regulação da bioatividade do $\mathrm{NO}$ e o aumento na capacidade de vasodilatação ${ }^{19}$. As adaptações decorrentes do estresse de cisalhamento no endotélio são transitórias e observadas na fase inicial do treinamento ${ }^{2,20}$. Em longo prazo ocorre uma adaptação estrutural ao treinamento, possivelmente devido ao remodelamento mediado pelo $\mathrm{NO}$, ou seja, aumento do calibre dos vasos ${ }^{2}$.

O treinamento físico pode contribuir para o relaxamento das células do músculo liso vascular. O treinamento aeróbico, por exemplo, promove aumento da complacência arterial e redução da pressão arterial sistólica ${ }^{17}$, já o efeito do treinamento resistido sobre a função vascular é controverso. Alguns estudos demonstraram que o treinamento resistido aumentou a rigidez arterial e reduziu a complacência arterial $^{21,22}$. Entretanto, outros estudos não observaram alteração nestes parâmetros ${ }^{23,24}$.

Quando o treinamento envolve exercícios com pequenos grupos musculares a melhora na função vasodilatadora dependente do endotélio é localizada, já os exercícios com grandes grupos musculares promovem alterações sistêmicas na pressão e frequência cardíaca, que geram o estresse de cisalhamento e, consequentemente, adaptações na função e estrutura vascular ${ }^{19}$. A intensidade do exercício pode influenciar na melhora da função vasodilatadora dependente do endotélio, visto que o exercício de intensidade baixa pode melhorar a função vascular mediada pelo NO e o exercício moderado aumenta a bioatividade de NO endotelial, enquanto o exercício de intensidade alta pode prejudicar a produção de NO, por este ser anulado pela produção de $\mathrm{RL}^{2}$. Entretanto, ao relacionar a capacidade oxidante com o exercício agudo de intensidades moderada e alta, observou-se que o exercício intervalado de alta intensidade foi mais eficaz em melhorar a capacidade antioxidante em indivíduos saudáveis, podendo-se especular que o maior estresse de cisalhamento gerado durante a sessão de exercício produziu maior resposta a nível celular e molecular ${ }^{25}$. O efeito protetivo do exercício sobre o sistema vascular é dependente da intensidade do exercício e está relacionado com a capacidade antioxidante induzida pelo exercício e, desta forma, sugere-se uma ligação com a biodisponibilidade de $\mathrm{NO}^{25}$.

Visto que no treinamento físico os diferentes volumes de massa muscular ou intensidade de exercício podem estar associados com diferentes impactos mediados pelo estresse de cisalhamento na função vascular ${ }^{26}$, assim como os tipos de exercício, aeróbico e resistido, podem promover diferentes adaptações na função $v_{\text {vascular }}{ }^{27}$, esta revisão sistemática tem como objetivo examinar evidências de que o treinamento com diferentes tipos de exercício influenciam o papel do NO derivado do endotélio na função vascular em indivíduos saudáveis. Este estudo poderá facilitar a elaboração de programas de treinamento que promova benefícios para a função vascular.

\section{MÉTODOS}

\section{Estratégias de Pesquisa}

Para realização desse estudo optou-se por uma revisão sistemática buscando artigos originais publicados entre janeiro de 1997 e maio de 2012, que abordaram o efeito do treinamento sobre a função vascular mediada pelo $\mathrm{NO}$ em indivíduos saudáveis. A pesquisa foi realizada nas bases de dados Medline e Science Direct utilizando-se associações dos seguintes termos indexadores: nitric oxide, vascular, endothelial adaptations, endothelium-dependent, exercise e training. Na base Medline 
foram utilizados os seguintes limites: estudos publicados entre 1997 e Maio de 2012, palavras no titlelabstract, humans, free full text, original articles, escritos em Inglês. Na base Science Direct os limites estabelecidos foram: publicados entre 1997 e Maio de 2012, somente original articles, palavras no title/abstract/key-words, somente em periodicos. Cabe ressaltar que em ambas as bases de dados, o termo "vascular" foi limitado somente no title.

\section{Critérios de inclusão e exclusão}

Para inclusão do artigo era necessário que fosse um estudo original, apresentando os efeitos crônicos do treinamento (resistido ou aeróbico) com humanos. Os critérios de exclusão empregados foram: 1) estudos apresentando somente o efeito agudo do exercício; 2) artigos sem detalhamento do programa de treinamento; 3) uso de drogas durante o tempo de treinamento; 4) pessoas com qualquer tipo de enfermidades.

Todos os artigos encontrados passaram inicialmente pelos autores, que realizaram a leitura do título para verificar se atendiam aos objetivos do estudo; no segundo momento foi realizada a leitura do resumo do artigo para verificar se atendiam aos critérios de inclusão e exclusão do presente estudo; e no último momento os artigos selecionados foram lidos na íntegra e posteriormente analisados para a extração dos dados. O fluxograma do processo de busca e seleção dos artigos encontrados está apresentado na figura 1.

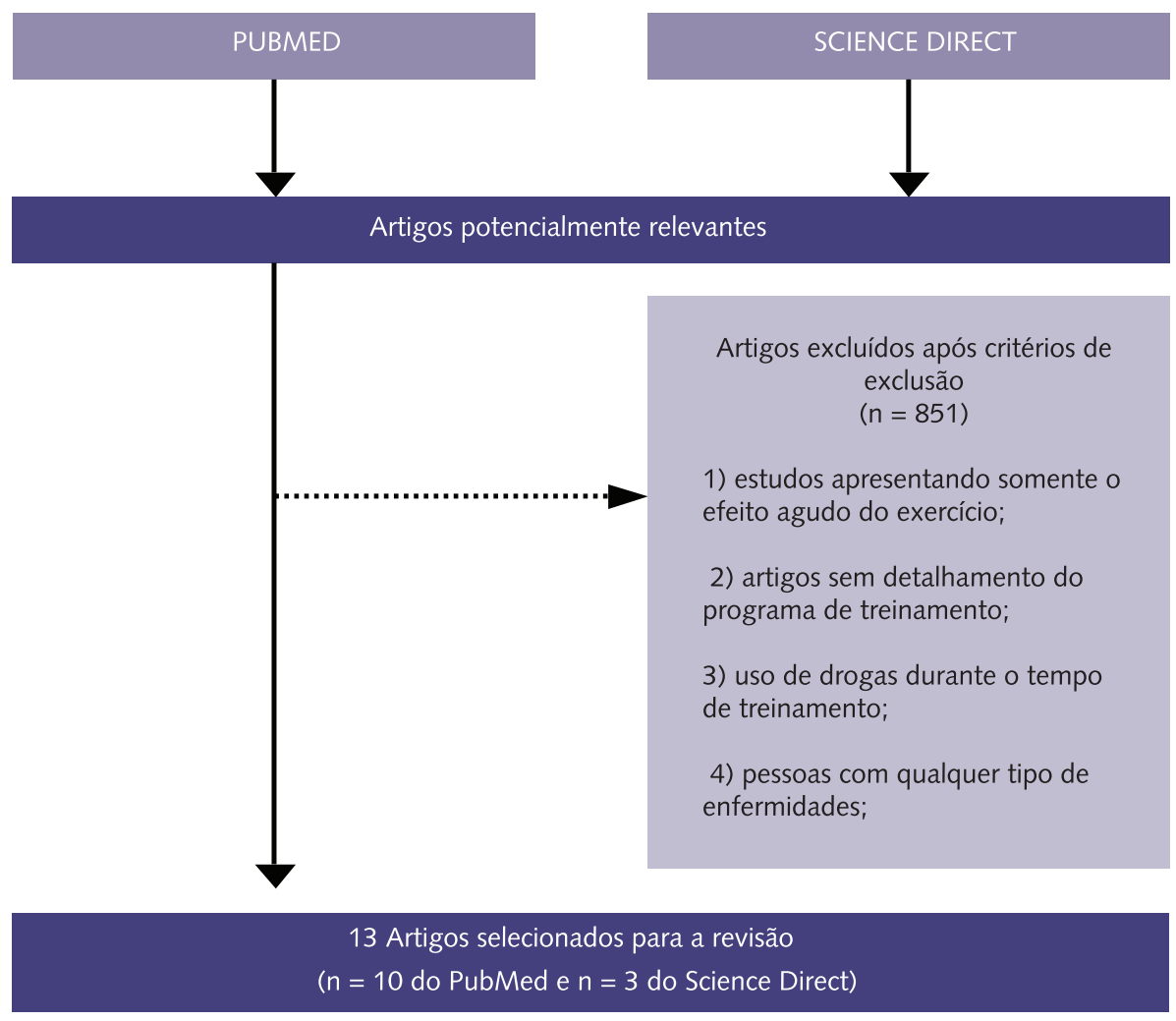

Figura 1 - Fluxograma do processo de seleção dos artigos.

\section{Análise dos dados}

$\mathrm{Na}$ análise dos artigos procurou-se extrair dados como: a população alvo, a faixa etária, o tipo de exercício, a frequência, a intensidade, a duração, o período, e os efeitos encontrados. Para análise os estudos foram separados segundo o tipo de intervenção: exercícios resistidos, aeróbicos ou combinados (resistido e aeróbico). 


\section{RESULTADOS}

Foram selecionados treze artigos após verificação dos critérios de inclusão e exclusão, sendo cinco estudos com treinamento usando exercício resistido ${ }^{28-32}$, sete com treinamento usando exercício aeróbico ${ }^{33-39}$ e um com ambos os tipos de exercício ${ }^{27}$. Os estudos selecionados apresentaram pesquisas com sujeitos do gênero masculino $^{21,29,31,32,35-37}$, feminino ${ }^{38}$ ou ambos os gêneros ${ }^{27,28,30,34,39}$, com a faixa etária variando entre 18 e 70 anos. Os resultados foram apresentados segundo o tipo de exercício usado no programa de treinamento (resistido, aeróbico ou combinado), a frequência (entre 2 e 7 sessões por semana), a intensidade (variada), a duração (variada) e o período (de 4 a 13 semanas), juntamente com os efeitos encontrados nos sujeitos.

O método utilizado para prescrever a intensidade do exercício variou entre os artigos. Dentre os estudos com exercício resistido foram utilizados percentuais de força de preensão manual máxima ${ }^{28,32}$, contração voluntária máxima ${ }^{29,31}$ e repetição máxima ${ }^{30}$; nos estudos com exercício aeróbico foram utilizados percentuais do consumo máximo de oxigênio $\left(\mathrm{VO}_{2 \max }\right)^{33,34,36,37}$, frequência cardíaca máxima ${ }^{35,39} \mathrm{e}$ limiar ventilatório individual ${ }^{37}$; e no estudo com exercícios combinados a intensidade foi apresentada em percentuais da frequência cardíaca de treino e repetição máxima ${ }^{27}$. A duração do exercício mudou conforme o tipo de exercício, no treinamento aeróbico foi apresentado em minutos (entre 30 e 60 minutos) ${ }^{33-39}$; o treinamento resistido foi apresentado em minutos (de 20 a 30) $)^{28,31,32}$, ciclo de contração-relaxamento (1,5 segundos $)^{29}$ e número de repetições (pelo menos 12$)^{30}$. Quando combinado o treinamento aeróbico e resistido, a duração foi 20 minutos para o aeróbico e 5 séries de 8-10 repetições para o resistido ${ }^{27}$.

Dos cinco estudos que utilizaram o treinamento resistido, quatro utilizaram treinamento isométrico ${ }^{28,29,31,32}$ e somente um realizou treinamento dinâmico ${ }^{30}$; nos estudos com treinamento aeróbico, três utilizaram o ciclo ergométrico para o exercício ${ }^{36-38}$, dois utilizaram a caminhada ${ }^{34,35}$, um utilizou corrida ${ }^{33}$ e um realizou caminhada e corrida no mesmo estudo ${ }^{39}$. O único estudo com treinamento combinado utilizou corrida e exercícios em aparelhos de musculação, com dois protocolos (um com exercício resistido realizado antes do aeróbico, e outro com aeróbico depois do exercício resistido) ${ }^{27}$.

A maioria dos estudos utilizou infusão de drogas para avaliar a função vascular. A acetilcolina foi utilizada para verificar a vasodilatação mediada pelo $\mathrm{NO}^{28,29,33-}$ 36; o nitroprussiato de sódio foi utilizado para verificar a vasodilatação independente do endotélio ${ }^{28,29,33,35}$; a N-monometil-L-arginina foi utilizada para inibir a $\mathrm{eNOS}^{38,33,34,36,39}$; e o dinitrato de isosorbida foi utilizado para verificar a vasodilatação independente do endotélio ${ }^{34}$. Os estudos que não utilizaram infusão de drogas mensuraram a produto final estável de óxido nítrico, ou seja, nitrito/nitrato (NOx) e endotelina-1 (ET-1) do plasma sanguíneo ${ }^{37,38}$ e analisaram o fluxo sanguíneo e a resistência vascular ${ }^{27,30-32}$.

Nos treinamentos com exercícios aeróbicos contínuos, foram observados aumentos no fluxo sanguíneo ${ }^{34-36}$ e na concentração de nitrito/nitrato ${ }^{37,38}$, e melhora na condutância ${ }^{35}$ e no tônus vascular ${ }^{39}$. No estudo de Bergolm et al. ${ }^{33}$ com treinamento de intensidade moderada a alta $\left(70-80 \mathrm{VO}_{2 \text { máx }}\right)$ a vasodilatação dependente do endotélio foi comprometida, enquanto no estudo realizado por Goto et al. ${ }^{36}$ houve resultado positivo no grupo de treinamento de intensidade moderada (50\% $\left.\mathrm{VO}_{2 \max }\right)$, porém não houve alteração nos grupos de intensidades baixa (25\% VO-

$\left.{ }_{2 \max }\right)$ e alta $\left(75 \% \mathrm{VO}_{2 \max }\right)$, sendo que na intensidade alta houve um aumento no estresse oxidativo (Tabela 1 ). 
Tabela 1 - Efeitos do treinamento aeróbico sobre a função vascular mediada pelo óxido nítrico.

\begin{tabular}{|c|c|c|c|c|c|c|c|c|}
\hline Estudo & Indivíduos & $\begin{array}{l}\text { Idade } \\
\text { (anos) }\end{array}$ & $\begin{array}{l}\text { Tipo de } \\
\text { Exercicio }\end{array}$ & $\begin{array}{c}\text { Freqüência } \\
\text { (sessões/sem.) }\end{array}$ & Intensidade & $\begin{array}{c}\text { Duração } \\
\text { (minutos) }\end{array}$ & $\begin{array}{c}\text { Período } \\
\text { (semanas) }\end{array}$ & Efeitos \\
\hline $\begin{array}{l}\text { Bergholm } \\
\text { et al. }{ }^{18}\end{array}$ & $9 \mathrm{H}$ & $26 \pm 1$ & Corrida & 4 & $\begin{array}{c}70-80 \% \text { do } \\
\mathrm{VO}_{2 \max }\end{array}$ & 60 & 12 & $\begin{array}{l}\leftrightarrow \text { do FS do braço } \\
\text { controle (infusão de } \\
\text { drogas vasodilatadoras) } \\
\downarrow \text { do FS dependente do } \\
\text { endotélio (infusão de } \\
\text { doses de ACh) } \\
\downarrow \text { do \% do FS dependente } \\
\text { do endotélio ( } \downarrow \text { \% no } \\
\text { fluxo basal por L-NMMA) } \\
\downarrow \text { FS basal antes da } \\
\text { infusão das drogas } \\
\leftrightarrow \text { do FS hiperêmico } \\
\text { reativo }\end{array}$ \\
\hline $\begin{array}{l}\text { Higashi et } \\
\text { al. }{ }^{19}\end{array}$ & $\begin{array}{l}5 \mathrm{H}, 1 \mathrm{M} \\
\text { (grupo } \\
\text { saudável- } \\
\text { exercitado) }\end{array}$ & $27 \pm 4$ & Caminhada & $5-7$ & $\begin{array}{l}52 \pm 9 \% \text { do } \\
\mathrm{VO}_{2 \max }\end{array}$ & 30 & 12 & $\begin{array}{l}\leftrightarrow \text { FS basal } \\
\uparrow \text { FS com ACh em treinados } \\
\leftrightarrow \text { com dinitrato de } \\
\text { isosorbida } \\
\text { Infusão de } L-N M M A \downarrow \text { a } \\
\text { resposta para } A C h\end{array}$ \\
\hline $\begin{array}{l}\text { De Souza } \\
\text { et al. }{ }^{20}\end{array}$ & $13 \mathrm{H}$ & $56 \pm 2$ & Caminhada & $\begin{array}{c}\text { 3-4 (3 semanas } \\
\text { iniciais) } \\
5-6 \\
\text { (posteriormante) }\end{array}$ & $\begin{array}{c}60 \% \text { FCM } \\
70-75 \% \text { FCM }\end{array}$ & $\begin{array}{c}30 \\
40-45\end{array}$ & 12 & $\begin{array}{l}\leftrightarrow \mathrm{FS} \text { e CV do antebraço } \\
\text { no repouso } \\
\uparrow \mathrm{FS} \text { e CV do antebraço } \\
\text { em resposta a ACh com } \\
\text { treinamento } \\
\leftrightarrow \text { na resposta ao NSP }\end{array}$ \\
\hline $\begin{array}{l}\text { Goto et } \\
\text { al. }{ }^{21}\end{array}$ & $26 \mathrm{H}$ & $25 \pm 2,5$ & $\begin{array}{c}\text { Ciclo } \\
\text { Ergômetro }\end{array}$ & $5-7$ & $\begin{array}{c}25,50 \text { e } 75 \% \\
\text { do } \mathrm{VO}_{2 \max }\end{array}$ & 30 & 12 & $\begin{array}{l}\uparrow \text { na resposta do FS } \\
\text { do antebraço ao ACh } \\
\text { no grupo de exercício } \\
\text { moderado e } \leftrightarrow \text { nos grupos } \\
\text { de baixa e alta intensidade } \\
\uparrow \text { do FS no antebraço } \\
\text { após infusão de DVIE nos } \\
\text { três grupos } \\
\downarrow \text { do FS no antebraço e } \\
\text { na resposta ao ACh após } \\
\text { infusão de L-NMMA nos } \\
\text { três grupos }\end{array}$ \\
\hline $\begin{array}{l}\text { Maeda et } \\
\text { al. } 22\end{array}$ & $8 \mathrm{H}$ & $\begin{array}{c}20,3 \pm \\
0,5\end{array}$ & $\begin{array}{c}\text { Ciclo } \\
\text { Ergômetro }\end{array}$ & $3-4$ & $\begin{array}{l}70 \% \text { do } \\
\mathrm{VO}_{2 \max }\end{array}$ & 60 & 8 & $\begin{array}{l}\uparrow \text { na concentração de } \\
\text { nitrito/nitrato (NOx) } \\
\text { no plasma após } 4 \text { e } 8 \\
\text { semanas de treinamento } \\
\downarrow \text { de ET-1 no plasma } \\
\text { após } 4 \text { e } 8 \text { semanas de } \\
\text { treinamento }\end{array}$ \\
\hline $\begin{array}{l}\text { Maeda et } \\
\text { al. }{ }^{23}\end{array}$ & $15 M$ & $56-69$ & $\begin{array}{c}\text { Ciclo } \\
\text { Ergômetro }\end{array}$ & 5 & $80 \%$ do LVI & 30 & 12 & $\begin{array}{l}\uparrow \text { da concentração de } \\
\text { NOx no plasma } \\
\uparrow \text { da concentração de } \\
\text { GMPc no plasma }\end{array}$ \\
\hline $\begin{array}{l}\text { Sugawara } \\
\text { et al. }{ }^{24}\end{array}$ & $2 \mathrm{H}, 5 \mathrm{M}$ & $60 \pm 3$ & $\begin{array}{l}\text { Caminhar/ } \\
\text { correr }\end{array}$ & $3-4$ & $65-75 \%$ FCM & $30-45$ & 12 & $\begin{array}{c}\leftrightarrow \text { FS da artéria femoral } \\
\uparrow \text { tônus vascular sistêmico } \\
\text { e local mediado pelo NO } \\
\uparrow \text { tônus vascular simpático } \\
\text { sistêmico e local (refletido } \\
\text { pelo } \uparrow \text { CV femoral com } \\
\text { bloqueio a-adrenergico) }\end{array}$ \\
\hline
\end{tabular}

$\mathrm{H}$ : homem; $\mathrm{M}$ : mulher; $\mathrm{VO}_{2 \max }$ : consumo máximo de oxigênio; FS: Fluxo sanguíneo; ACh: Acetilcolina; L-NMMA: N-monometil-L-arginina; FCM: Freqüência cardíaca máxima; CV: condutância vascular; NSP: Nitroprusssiato de sódio; DVIE: Dinitrato vasodilatador independente do endotélio; NOx: Produto final estável de óxido nítrico (nitrito/nitrato); ET-1: Endotelina-1; LVI: Limiar ventilatório individual; GMPc: Monofosfato de guanosina cíclico; NO: óxido nítrico. 
Nos treinamentos com exercícios resistidos, observaram-se aumentos no fluxo sanguíneo ${ }^{28-32}$ e na condutância vascular ${ }^{28,29}$. No estudo de Franke et al. ${ }^{29}$ com exercício isométrico de intensidade alta ( $70 \%$ da contração voluntária máxima), apesar dos aumentos no fluxo sanguíneo e na condutância vascular máxima após o treinamento resistido, a liberação de NO (estimulada pela acetilcolina) não foi diferente antes e após o período de treinamento. Além disso, os autores não encontraram alterações na sensibilidade do músculo liso aos vasodilatadores liberados, avaliados através do nitroprussiato de sódio (Tabela 2).

No estudo de Okamoto et al. ${ }^{27}$ com treinamento combinado (aeróbico contínuo e resistido), observou-se que apenas quando o exercício aeróbico foi realizado após o resistido houve efeito positivo, tais como aumento no fluxo sanguíneo e redução na rigidez arterial e na velocidade de onda de pulso (Tabela 3).

Tabela 2 - Efeito do treinamento resistido sobre o óxido nítrico em pessoas saudáveis.

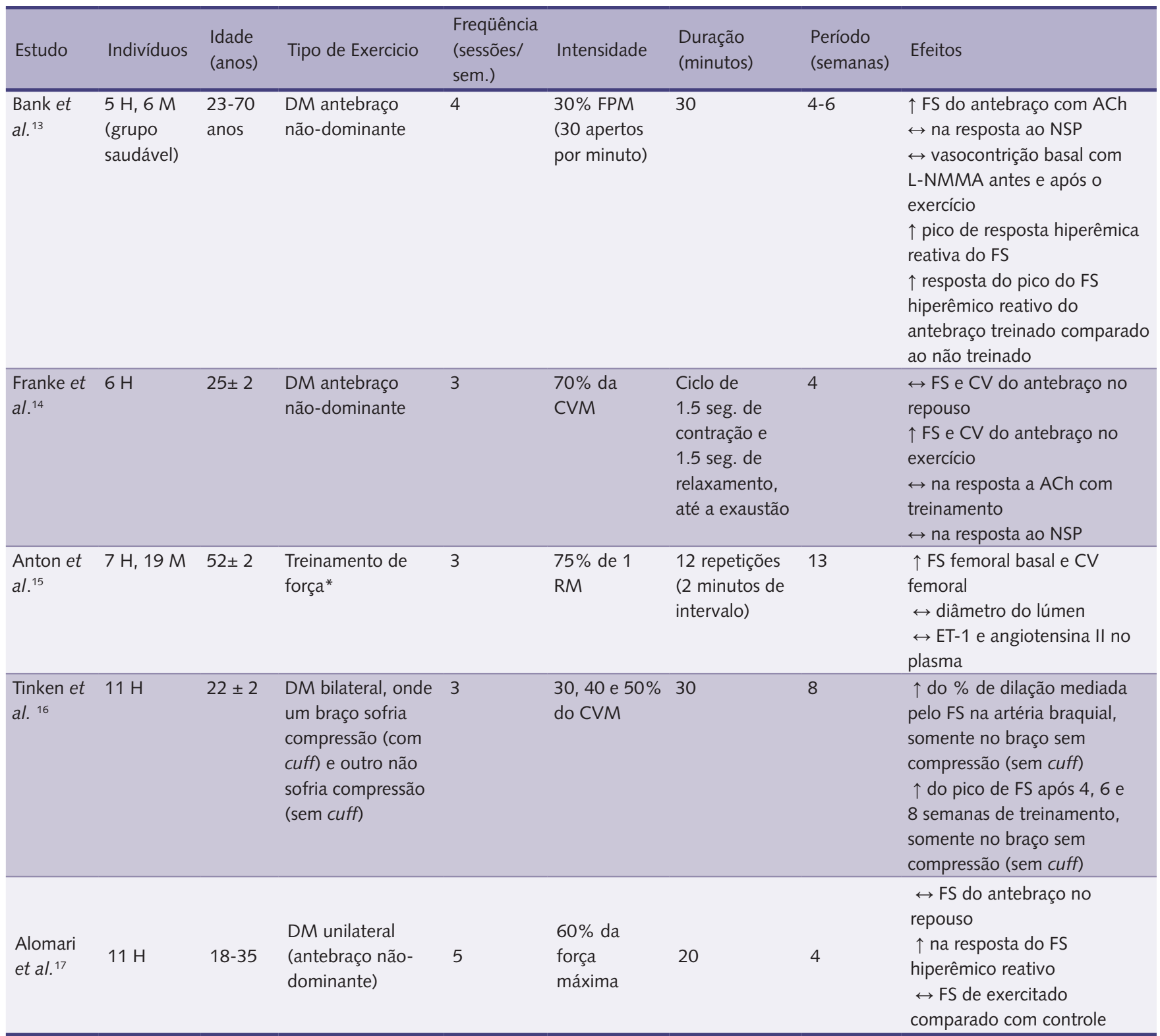

H: homem; M: mulher; DM: dinamometria manual; FPM: Força de preensão manual máxima; FS: fluxo sanguíneo; ACh: Acetilcolina; NSP: Nitroprusssiato de sódio; L-NMMA: N-monometil-L-arginina; CVM: Contração voluntária máxima; CV: condutância vascular; RM: repetição máxima; ET-1: Endotelina. * peito, leg press bilateral, costa, ombros, tríceps, bíceps, panturrilha e abdômen. 
Tabela 3 - Efeito do treinamento aeróbico e resistido sobre o óxido nítrico em pessoas saudáveis.

\begin{tabular}{|c|c|c|c|c|c|c|c|c|}
\hline Estudo & Indivíduos & $\begin{array}{l}\text { Idade } \\
\text { (anos) }\end{array}$ & Tipo de Exercicio & $\begin{array}{l}\text { Freqüência } \\
\text { (sessões/ } \\
\text { sem.) }\end{array}$ & Intensidade & $\begin{array}{l}\text { Duração } \\
\text { (minutos) }\end{array}$ & $\begin{array}{l}\text { Período } \\
\text { (semanas) }\end{array}$ & Efeitos \\
\hline
\end{tabular}

H: homem; M: mulher; FCT: freqüência cardíaca de treino; RM: repetição máxima; FS: fluxo sanguíneo.

\section{DISCUSSÃO}

A maioria dos estudos desta revisão apresentou resultados positivos do treinamento sobre a função vascular mediada pelo NO. Os principais achados foram: 1) os treinamentos com exercícios aeróbicos contínuos e resistidos melhoram a função vascular mediada pelo NO;2) os treinamentos com exercícios aeróbicos contínuos e resistidos de intensidade alta não alteram ou comprometem a vasodilatação dependente do endotélio; 3 ) no treinamento combinado, quando o exercício resistido foi realizado após o exercício aeróbico não houve melhora na função vascular.

Entre os estudos analisados, existem certos pontos que merecem uma atenção especial. Um dos mais importantes achados foi que os treinamentos de intensidade alta (aeróbico e resistido) ${ }^{29,33,36}$ não proporcionaram efeitos positivos sobre a função vascular mediada pelo NO. No treinamento com exercício resistido de intensidade alta, os aumentos no fluxo sanguíneo e na condutância vascular durante o exercício, induzidos pela administração de acetilcolina, foram semelhantes antes e após o período de treinamento, o que levou os autores a defenderem que o treinamento resistido de intensidade alta não potencializa a vasodilatação mediada pelo $\mathrm{NO}^{29}$.

Nos estudos que usaram treinamento com exercícios aeróbicos contínuos por doze semanas, com intensidade moderada para alta, foi observado comprometimento na vasodilatação dependente do endotélio, o que poderia ser uma resposta à redução dos antioxidantes em detrimento da intensidade alta $^{33}$, e com alta intensidade foi observado aumento no estresse oxidativo ${ }^{36}$. Estes resultados confirmariam o que Green $e t a l^{2}$ defendem, que o exercício de alta intensidade pode prejudicar a vasodilatação dependente do endotélio pelo aumento de RL, o que compromete a produção de NO. Entretanto, em um estudo com indíviduos saudáveis, o exercício agudo intervalado de alta intensidade aumentou a dilatação mediada pelo fluxo sanguíneo e a capacidade antioxidante ${ }^{25}$. Em outro estudo, também foi observado que o treinamento intervalado de alta intensidade melhorou a rigidez arterial periférica e a dilatação mediada pelo fluxo sanguíneo em indivíduos saudáveis ${ }^{40}$.

Para Tjonna et al. ${ }^{41} \mathrm{o}$ estresse de cisalhamento gerado nos programas de treinamento de intensidades baixa e alta afetaria de forma distinta os vasos sanguíneos durante o treinamento, o que proporcionaria diferente resposta molecular à 
intensidade adotado no treinamento. $\mathrm{O}$ modelo de treinamento intervalado de alta intensidade, diferente do treinamento contínuo, permite períodos de repouso que possibilita ao indivíduo completar um curto período de trabalho intenso, que, deste modo, desafia a capacidade de bombeamento do coração mais do que seria possível em menores intensidades ${ }^{41}$.

Quanto ao estudo com treinamento aeróbico contínuo que apresentou redução na função vascular dependente do endotélio ${ }^{33}$, vários fatores podem ter influenciado os achados, como a metodologia empregada para avaliar a função endotelial, os sujeitos avaliados, o tipo de exercício adotado, a frequência e a duração do treinamento. Desse modo, mais estudos sobre os efeitos do treinamento de intensidade alta na função vascular dependente do endotélio devem ser realizados para fortalecer os resultados encontrados.

Com relação ao tamanho da massa muscular envolvida no exercício, os estudos demonstram diferentes respostas ao utilizar pequenos e grandes grupos musculares ${ }^{19,43}$. Nesta revisão os estudos com treinamento aeróbico (tabela 1) utilizaram apenas grandes grupamentos musculares, não sendo possível identificar os efeitos do treinamento aeróbico envolvendo pequenos grupos musculares na resposta da função vascular mediada pelo NO, desta forma estudos que utilizam treinamento aeróbico com pequenos grupos musculares são necessários. Com relação ao treinamento resistido (tabela 2) a maioria utilizou pequenos grupos musculares e apresentaram resultados positivos, o único estudo com grandes grupamentos musculares $^{30}$ também apresentou resultados positivos, porém, mais estudos são necessários para confirmar o efeito do treinamento resistido na função vascular quando se utiliza grandes grupamentos musculares.

Estudos que usaram treinamentos com exercícios resistidos têm demonstrado redução na função vascular ${ }^{21,22,42}$. Uma possível justificativa é que o aumento agudo intermitente da pressão arterial na região cardiotorácica durante o exercício pode resultar em um aumento crônico do conteúdo muscular liso da parede arterial e nas propriedades de que sustentam a carga exercida pelo fluxo sanguíneo decorrente do exercício, que são o colágeno e a elastina, o que diminuiria a complacência $\operatorname{arterial}^{22}$. Apesar da controversa quanto ao benefício do treinamento com exercícios resistidos, os resultados encontrados nesta revisão demonstram que a utilização deste treinamento proporcionou efeitos positivos sobre a função vascular mediada pelo NO. Nos estudos de Rakobowchuk et al. ${ }^{23,24}$ também foram encontrados resultados positivos do treinamento resistido de doze semanas em jovens saudáveis que não apresentaram alterações na rigidez e na complacência arterial ${ }^{24}$; e apresentaram aumento no diâmetro arterial braquial em repouso, sendo uma justificativa para este resultado o estresse de cisalhamento transitório pós-exercí$\mathrm{Cio}^{23}$. É importante destacar que dentre os estudos que usaram treinamentos com exercícios resistidos abordados nesta revisão, nenhum verificou os efeitos deste tipo de treinamento sobre a rigidez e a complacência arterial.

Quando os tipos de exercícios foram combinados (tabela 3), apenas no grupo em que o treinamento aeróbico foi realizado após o resistido houve uma melhora na função vascular mediada pelo $\mathrm{NO}^{27}$, não havendo diferenças quando o exercício aeróbico foi realizado antes do resistido. $\mathrm{O}$ autor argumenta que apesar do exercício aeróbico apresentar resultados positivos sobre a função vascular, estes efeitos podem ser neutralizados pelo treinamento resistido posterior, enquanto o exercício aeróbico após o resistido neutraliza os efeitos negativos deste último.

Esta parece ser uma maneira eficaz de combinação dos tipos de exercício em um treinamento para maximizar os benefícios e minimizar os malefícios de de- 
terminado tipo de exercício. Porém, cabe ressaltar a necessidade de mais estudos abordando o treinamento combinado (aeróbico e resistido) a fim de confirmar os resultados encontrados. Além disso, deve-se estabelecer os grupamentos musculares, a intensidade e a duração do treinamento para determinar qual a estratégia ideal a ser adotada para a melhora da função vasodilatadora.

Nos estudos revisados, os sujeitos apresentaram uma ampla faixa etária (18-70 anos), sendo que nos cinco estudos, com indivíduos acima de 50 anos de ida$\mathrm{de}^{28,30,35,38,39}$, observaram-se resultados positivos. O estudo de Sugawara et al..$^{39} \mathrm{com}$ sujeitos de meia idade e idosos encontrou aumento do tônus vascular local e sistêmico mediado pelo NO, porém, não houve alteração no fluxo sanguíneo, sendo este devido ao efeito concorrente da atividade do sistema nervoso simpático elevada e maior biodisponibilidade do NO sobre a vasculatura.

$\mathrm{O}$ fator idade pode ter influenciado nos resultados encontrados, pois com o envelhecimento há uma diminuição na complacência arterial devido às alterações estruturais (como aumento do colágeno) e funcionais (como redução da biodisponibilidade do $\mathrm{NO})^{44}$. Neste sentido, a prática de exercício aeróbico regular mostra-se uma estratégia atraente para melhorar a rigidez arterial em indivíduos de meia idade e idosos sedentários ${ }^{44,45}$. Porém, diferentemente dos estudos anteriores que defendem que somente o treinamento aeróbico pode atenuar o declínio ocorrido com a idade e melhorar a complacência arterial, no estudo de Anton et al. ${ }^{30}$ o treinamento com exercício resistido com duração de treze semanas aumentou o fluxo sanguíneo e a condutância vascular femoral basal em indivíduos de meia-idade e idosos.

A maioria dos estudos encontrados utilizou a infusão de drogas para verificar a função vascular $28,29,33-36,39$. Cabe ressaltar que a infusão de fármacos na artéria braquial permite o estudo direto dos efeitos vasculares de determinada droga ${ }^{46}$. Alguns estudos utilizaram a medida do fluxo sanguíneo e resistência vascular ${ }^{27,30-32}$, pois sabe-se que o aumento do fluxo sanguíneo gera um estresse de cisalhamento, que estimula o endotélio a liberar $\mathrm{NO}^{18}$. Porém, Anton et al. ${ }^{30}$ e Alomari et al. ${ }^{32}$ ressaltam a necessidade de mais estudos para avaliar os mecanismos específicos envolvidos nas mudanças vasculares.

Os resultados desta revisão apontam que o treinamento físico exerce um papel importante sobre a melhora na função vascular mediada pelo $\mathrm{NO}$ em população saudável, sendo a estratégia adotada fundamental para que a melhora seja efetiva. Sendo assim, ao elaborar um programa de treinamento que vise melhora na função vascular é importante considerar a intensidade que será adotada nas sessões de treinamento, o grupamento muscular envolvido nos exercícios propostos e a combinação dos tipos de exercício.

Mais estudos com o treinamento combinado são necessários a fim de confirmar os resultados encontrados até o momento. É importante normalizar as metodologias empregadas nos estudos, pois possibilita uma melhor comparação entre os mesmos. Além disso, mais estudos randomizados controlados são necessários a fim de estabelecer comparações entre grupos.

Em conclusão, o treinamento físico com exercícios aeróbico ou resistido influencia beneficamente o papel do NO derivado do endotélio na função vascular. O treinamento de alta intensidade, com exercícios aeróbicos contínuos ou resistidos, pode atenuar os benefícios do exercício sobre a função vascular. Porém, é importante ressaltar a necessidade de mais estudos, visto que vários fatores podem interferir nos resultados, e que outros estudos, utilizando o treinamento intervalado de alta intensidade, não apresentaram resultados semelhantes. 


\section{Agradecimentos}

AJ Natali é bolsista de produtividade do CNPq.

\section{Contribuição dos autores}

Rodrigues AC e Oliveira RAR realizaram a busca, a seleção, a análise dos artigos e a elaboração do artigo. Marins JCB e Natali AJ revisaram e corrigiram a versão final do manuscrito. Todos os autores contribuíram significativamente para assumirem autoria pelo estudo.

\section{REFERÊNCIAS}

1. Totzeck M,Hendgen-Cotta UB, Rammos C, et al. Higher endogenous nitrite levels are associated with superior exercise capacity in highly trained athletes. Nitric Oxide 2012; 27: 75-81.

2. Green DJ, Maiorana A, O’Driscoll G, Taylor R. Effect of exercise training on endotheliumderived nitric oxide function in humans. J Physiol 2004; 1-25.

3. Roberts CK, Barnard RJ, Jasman A, BalonTW. Acute exercise increases nitric oxide synthase activity in skeletal muscle. Am J Physiol Endocrinol Metab 1999; 390-394.

4. Stoner L, Erickson ML, Young JM, et al. There's more to flow-mediated dilation than nitric oxide. J Atheroscler Thromb 2012; 19: 000-000.

5. Valko M, Leibfritz D, Mancol J, et al. Free radicals and antioxidants in normal physiological functions and human disease. Int J Biochem Cell Biol 2007; 39(1): 44-84.

6. Furchgott RF, Zawadzki JV. The obligatory role of endothelial cells in the relaxation of arterial smooth muscle by acetylcholine. Nature 1980; 288: 373-376.

7. Ignarro LJ, Buga GM, Wood KS, et al. Endothelium-derived relaxing factor produced and released from artery and vein is nitric oxide. Proc Natl Acad Sci USA 1987; 84: 9265-9

8. Palmer RMJ, Ferrige AG, Moncada S. Nitric oxide release accounts for the biological activity of endothelium-derived relaxing factor. Nature, 1987; 524-6.

9. Powers SK, Jackson MJ. Exercise-Induced Oxidative Stress: Cellular Mechanisms and Impact on Muscle Force Production. Physiol Rev 2008, 88: 1243-1276.

10. Fukai T, Folz RJ, Landmessera U, Harrisona DG. Extracellular superoxide dismutase and cardiovascular disease. Cardiovasc Res 2002; 55(2): 239-249.

11. Rush JWE, Denniss SG, Graham DA. Vascular nitric oxide and oxidative stress: Determinants of endothelial adaptations to cardiovascular disease and to physical activity. Can J Appl Physiol 2005; 30(4): 442-474.

12. Pereira AC, Paulo M, Araújo AV, et al. Nitric oxide synthesis and biological functions of nitric oxide released from ruthenium compounds. Braz J Med Biol Res 2011; 44(9): 947-57.

13. Halliwell B. Free radicals and antioxidants - quo vadis?. Trends Pharmacol Sci 2011;32(3): 125-30

14. Gomes EC, Silva AN, Oliveira, MR. Oxidants, antioxidants, and the beneficial roles of exercise-induced production of reactive species. Oxid Med Cell Longev 2012.

15. Brooks SV, Vasilaki A, Larkin LM. Repeated bouts of aerobic exercise lead to reductions in skeletal muscle free radical generation and nuclear factor kappaB activation. J Physiol 2008; 586(16): 3979-90.

16. Drummond GR, Cai H, Davis ME, et al. Transcriptional and posttranscriptional regulation of endothelial nitric oxide synthase expression by hydrogen peroxide." Circ Res 2000; 86(3): 347-54.

17. Mcallister RM, Newcomer SC, Laughlin MH. Vascular nitric oxide: effects of exercise training in animals. Appl Physiol Nutr Metab 2008; 33(1): 173-8.

18. Pyke KE,Tschakovsky ME. The relationship between shear stress and flow-mediated dilatation: implications for the assessment of endothelial function. J Physiol 2005; 357-69.

19. Maiorana A, O’Driscoll G, Taylor R, Green D. Exercise and the Nitric Oxide Vasodilator System. Sports Med 2003; 33(14): 1013-35.

20. Tinken TM, Thijssen DH, Black MA, et al. Time course of change in vasodilator function and capacity in response to exercise training in humans. J Physiol 2008; 586(20): 5003-12.

21. Bertovic DA, Waddell TK, Gatzka CD, et al. Muscular strength training is associated with low arterial compliance and high pulse pressure. Hipertension 1999; 33: 1385-91.

22. Miyachi M, Donato AJ, Yamamoto K, et al. Greater Age-Related Reductions in Central Arterial Compliance in Resistance-Trained Men. Hypertension 2003; 41: 130-5.

23. Rakobowchuk M, McGowan CL, Groot PC, et al. Endothelial function of young healthy males following whole body resistance training. J Appl Physiol 2005; 98: 2185-2190.

24. Rakobowchuk M, Mcgowan CL, De Groot PC, et al. Effect of whole body resistance training on arterial compliance in young men. Exp Physiol 2005; 90(4): 645-51. 
25. Tyldum GA, Schjerve IE, Tjønna AE, et al. Endothelial dysfunction induced by post-prandial lipemia: complete protection afforded by high-intensity aerobic interval exercise. J Am Coll Cardiol 2009; 53(2): 200-6.

26. Thijssen DHJ, Maiorana AJ, O'Driscoll G, et al. Impact of inactivity and exercise on the vasculature in humans. Eur J Appl Physiol 2010; 108: 845-75.

27. Okamoto T, Masuhara M, Ikuta K. Combined aerobic and resistance training and vascular function: effect of aerobic exercise before and after resistance training. J Appl Physiol 2007; 103: $1655-61$.

28. Bank AJ, Shammas RA, Mullen K, Chuang PP. Effects of short-term forearm exercise training on resistance vessel endothelial function in normal subjects and patients with heart failure. Journal of Cardiac Failure 1998; 4(3): 193-201.

29. Franke WD, Stephens GM, Schmid PG. Effects of intense exercise training on endotheliumdependent exercise induced vasodilatation. Clin Physiol 1998; 18(6): 521-8.

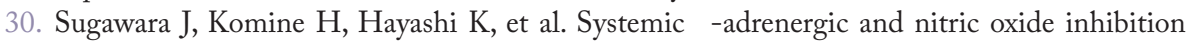
on basal limb blood flow: effects of endurance training in middle-aged and older adults. Am J Physiol Heart Circ Physiol 2007; 293: H1466-72.

31. Anton MM, Cortez-Cooper MY, De Van AE, et al. Resistance training increases basal limb blood flow and vascular conductance in aging humans. J Appl Physiol 2006; 101: 1351-55.

32. Tinken TM, Thijssen DHJ, Hopkins N, et al. Shear Stress Mediates Endothelial Adaptations to Exercise Training in Humans. J Hipertens 2010; 55: 312-18.

33. Alomari MA, Mekary RA, Welsch MA. Rapid vascular modifications to localized rhythmic handgrip training and detraining. Eur J Appl Physiol 2010; 109(5): 803-09.

34. Bergholm R, Makimattila S, Valkonen M, et al. Intense physical training decreases circulating antioxidants and endothelium-dependet vasodilation in vivo. Atherosclerosis 1999; 145: 341-49.

35. Higashi Y, Sasaki S, Kurisu S, et al. Regular aerobic exercise augments endothelium-dependent vascular relaxation in normotensive as well as hypertensive subjects. Circulation 1999; 100: 1194-2012.

36. De Souza CA, Shapiro LF, Clevenger CM, et al. Regular aerobic exercise prevents and restores age-related declines in endothelium-dependent vasodilation in healthy men. Circulation 2000; 102: 1351-57.

37. Goto C, Higashi Y, Kimura M, et al. Roles of endothelium-dependent nitric oxide and oxide stress. Circulation 2003; 108: 530-5.

38. Maeda S, Miyauchi T, Kakiyama T, et al. Effects of exercise training of 8 weeks and detraining on plasma levels of endothelium-derived factors, endothelin-1 and nitric oxide, in healthy young humans. Life Sciences 2001; 69: 1005-16.

39. Maeda S, Tanabe T, Otsuki T, et al. Moderate regular exercise increases basal production of nitric oxide in elderly women. Hipertens Res 2004; 27(12).

40. Rakobowchuk M, Tanguay S, Burgomaster KA, et al. Sprint interval and traditional endurance training induce similar improvements em peripheral arterial stiffness and flow-mediated dilation in healthy humans. Am J Physiol Regul Integr Comp Physiol 2008; 295: 236-42.

41. Tjonna AE, Lee SJ, Rognmo $\varnothing$, et al. Aerobic interval training versus continuous moderate exercise as a treatment for the metabolic syndrome: a pilot study. Circulation 2008; 118(4):346-54.

42. Miyachi M, Kawano H, Sugawara J, et al. Unfavorable effects of resistance training on central artery compliance: A randomized intervention study. Circulation 2004; 110(18): 2858-63.

43. Thijssen DHJ, Dawson EA, Black MA, et al. Brachial Artery Blood Flow Responses to Different Modalities of Lower Limb Exercise. Med Sci Sports Exerc 2009; 41(5): 1072-9.

44. Gates PE, Seals DR. Decline in large elastic artery compliance with age: a therapeutic target for habitual exercise. Br J Sports Med 2006; 40(11): 897-99.

45. Seals DR, De Souza CA, Donato AJ, Tanaka H. Habitual exercise and arterial aging. J Appl Physiol 2008; 105: 1323-32.

46. Benjamin N, Calver A, Collier J, et al. Measuring forearm blood flow and interpreting the responses to drugs and mediators. Hipertension 1995; 25: 918-23.

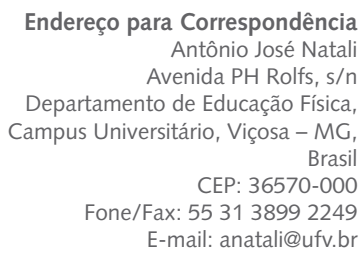

Recebido 15/09/12

Revisado 29/11/12

Aprovado 13/12/12 